\title{
Fatores Sócio-Demoğráficos e de Assistência Médica Associados ao Óbito Materno
}

\author{
Social, Demographic and Medical Care Factors Associated with Maternal Death
}

Rivaldo Mendes de Albuquerque ${ }^{1}$, José Guilherme Cecatti ${ }^{2,3}$

Ellen Hardy ${ }^{2,3}$, Aníbal Faúndes ${ }^{2,3}$

\begin{abstract}
RESUM0
O presente estudo avaliou os óbitos de mulheres com idade de 10 a 49 anos, ocorridos em Recife, Pernambuco, nos anos 1992 e 1993, com a finalidade de identificar os fatores sóciodemográficos, gestacionais e da assistência médica associados a esses óbitos maternos. Foram levantadas e revisadas 1.013 declarações de óbito, sendo identificados 42 casos de morte materna. Os dados desses óbitos foram complementados com informações clínicas, de necrópsias e de entrevistas com médicos dos hospitais onde ocorreram os óbitos e com familiares das mulheres falecidas. Quase dois terços (62\%) dos óbitos maternos ocorreram em mulheres de 20 a 29 anos de idade e mais da metade delas era solteira. Houve um maior número de óbitos nos partos cesarianos em relação aos vaginais. A maioria dos óbitos ocorreu nos três primeiros dias de hospitalização e aproximadamente 90\% das despesas hospitalares foram custeadas pelo Sistema Único de Saúde (SUS).
\end{abstract}

PALAVRAS-CHAVE: Mortalidade materna. Complicações da gestação. Aborto. Assistência médica.

\section{Introdução}

A morte tem sido uma das maiores interrogações na história da humanidade e representa um dos fenômenos que mais altera as relações sociais com que o homem se depara no seu cotidiano. Segundo Ariés ${ }^{2}$, em todo o Ocidente de cultura latina, católico ou protestante, a morte de um homem modifica solenemente o espaço e o

\footnotetext{
${ }^{1}$ Fundação Universidade de Pernambuco, Recife, PE

${ }^{2}$ Departamento de Tocoginecologia, Faculdade de Ciências Médicas, Universidade Estadual de Campinas, SP

${ }^{3}$ Centro de Pesquisas das Doenças Materno-Infantis de Campinas, CEMICAMP

Correspondência:

José Guilherme Cecatti

R. Edilberto Luiz Pereira da Silva, 296

13083-190 - Campinas - SP

Fax: 019-2893687
}

tempo de um grupo social, podendo se estender para uma comunidade inteira.

Quando uma mulher morre como conseqüência da gestação, parto ou puerpério, dever-se-ia interrogar onde começou a se construir esta morte. Qual a sua história de vida e a cadeia de eventos sociais e biológicos que levaram à morte? Sem dúvida, as disparidades na distribuição da renda e no acesso à infra-estrutura dos bens e serviços da sociedade estão diretamente relacionadas com a experiência de vida da população menos privilegiada e da mais favorecida, não permitindo que se efetive a igualdade das mulheres perante a morte, impedindo que a maioria possa ter uma morte "nãoantecipada", interrompendo suas vidas antes da hora, em plena capacidade reprodutiva e produtiva. Entretanto, uma abordagem como esta da 
morte materna não é tarefa fácil, considerando que em nosso meio até o conhecimento quantitativo sobre as mortes maternas está longe de ser completo. Os estudos sobre mortalidade materna, nos países em desenvolvimento, são geralmente descritivos, incluindo pequenas populações ou apenas estatísticas institucionais ${ }^{15}$. Entretanto, têm maior relevância aqueles de base populacional, já que os resultados melhor se aplicam à população de referência. Estes estudos são, contudo, ainda pouco freqüentes no país ${ }^{1,4,6,12,16}$.

Um estudo brasileiro de base populacional, a partir de variáveis sócio-demográficas e clínicas das mulheres que tiveram óbito materno e de um grupo controle de mulheres no ciclo gravídicopuerperal, procurou identificar possiveis fatores de risco para a ocorrência da morte materna. Concluiu que mulheres com patologias associadas à gestação detectadas no pré-natal e cujo parto ocorreu por cesárea, apresentaram maior risco de morrer $^{5}$. De fato, o parto por cesariana aparece de maneira consistente na literatura médica como associado ao óbito materno ${ }^{7,8,9,14}$.

No Brasil, e em especial no nordeste brasileiro, a mortalidade materna contribui como mais um fator de comprometimento da precária situação social, econômica e de saúde de seu povo, retirando precocemente a mulher do contato familiar e das atividades econômicas, desestruturando a família e transformando muitas crianças órfãs em menores de rua, fazendo-as enfrentar, além da perda da mãe, a marginalização a que são relegadas.

O desconhecimento do problema da mortalidade materna no nordeste brasileiro, aliado à necessidade de estabelecimento de metas prioritárias para a saúde das mulheres desta região, justifica seu estudo. O conhecimento da história das mortes maternas e dos fatores a elas associados deverá contribuir para que as autoridades sanitárias, a sociedade e as próprias mulheres exijam prioridade no atendimento integral à sua saúde, reconhecendo essa prática definitivamente como a mais efetiva para a promoção da saúde reprodutiva e para assegurar o seu direito à cidadania.

\section{Material e métodos}

Foram analisados os óbitos de mulheres de 10 a 49 anos de idade, residentes em Recife e que ocorreram neste município, no período de janeiro de 1992 a dezembro de 1993. Os dados foram obtidos a partir das declarações de óbito, dos prontuários médicos, de necrópsias e de entrevistas com médicos dos hospitais onde ocorreram os óbitos e com familiares das mulheres que faleceram. Informações detalhadas sobre material e métodos utilizados no estudo encontram-se disponíveis em outras publicações a ele referentes $^{1,6}$.

Para as variáveis relativas às características sócio-demográficas das mulheres, as diferenças entre as mortes maternas e as mortes de mulheres em idade fértil foram avaliadas através do cálculo do $\chi^{2}$ da distribuição. Para as variáveis relativas à características gestacionais e da assistência médica, avaliaram-se as distribuições percentuais das mulheres nas diferentes categorias.

\section{Resultados}

Foram identificados 1.013 óbitos de mulheres em idade fértil, de 10 a 49 anos, residentes no município de Recife e que faleceram nesta cidade, no período de 1 de janeiro de 1992 a 31 de dezembro de 1993. Somando os óbitos maternos declarados e os confirmados após investigação, identificaramse 42 mortes maternas. Quase dois terços (62\%) destes óbitos ocorreram na faixa etária de 20 a 29 anos. Na mortalidade geral em mulheres em idade fértil, esta faixa etária contribuiu com menos de $1 / 5$ do total das mortes. A diferença na distribuição etária entre os dois grupos foi altamente significativa. Mais da metade das mulheres no grupo de mortes maternas eram solteiras, mas foi elevado o número de mulheres sobre as quais não se conhecia o estado civil e por isso as diferenças não foram significantes (Tabela 1).

Quanto à ocupação, 81\% das mulheres que faleceram por complicações da gravidez, parto ou puerpério não exerciam atividade remunerada segundo a declaração de óbito, porcentual maior que o da população feminina de 10 a 49 anos (62\%). Esta diferença não foi estatisticamente significativa. Aproximadamente $3 / 4$ dos atestados não registravam o grau de instrução, o que impossibilitou o cálculo da significância estatística das diferenças encontradas (Tabela 1). Quase dois terços (61\%) das mulheres fizeram alguma consulta pré-natal.

A Tabela 2 mostra os antecedentes obstétricos das mulheres com morte materna, com relação à paridade, número de abortos e cesáreas. Quase a metade (47\%) destas mulheres tinha um ou dois filhos e a grande maioria não tinha antecedente de aborto (91\%), nem de cesárea (88\%).

Observa-se que quase a metade (44\%) das mortes maternas ocorreu com idade gestacional a partir de 37 semanas, ou no termo (Tabela 3). A gravidez terminou em parto em mais da metade (57\%) dos casos, 54\% dos quais por cesárea. Quase 
um terço $(30 \%)$ das mulheres faleceram ainda grávidas e 11,9\% das mortes maternas ocorreram por aborto induzido (Tabela 4). Todos os partos, e também os óbitos, ocorreram em hospitais e identificou-se um caso de aborto realizado no "consultório" de um auxiliar de enfermagem. Duas mulheres submeteram-se a procedimentos cirúrgicos não-obstétricos: um caso de drenagem de abscesso mamário que evoluiu para quadro séptico e outro de colecistectomia por empiema da vesícula biliar.

A maioria dos óbitos (65\%) ocorreu dentro dos três primeiros dias de internação sendo que 16\% das mulheres haviam sido transferidas de outro hospital. Na grande maioria dos óbitos, as despesas médico-hospitalares foram custeadas pelo Sistema Único de Saúde (SUS) (Tabela 5). Mais da metade $(52,3 \%)$ dos óbitos ocorreu no puerpério e aproximadamente um terço aconteceu em mulheres ainda gestantes. Apenas cinco mortes ocorreram após um aborto (Tabela 6).

Em relação à sobrevida dos recém-nascidos, pode-se observar que nove de cada dez sobreviveram à morte das suas mães (Tabela 7). Aproximadamente dois terços das mulheres que faleceram tiveram algum tipo de atendimento prénatal, sem levar em consideração nenhum critério quantitativo ou qualitativo das consultas.

Tabela 1 - Características sócio-demográficas das mulheres com óbito em idade fértil. Recife, 1992-1993 (em porcentagem).

\begin{tabular}{rcc}
\multicolumn{1}{l}{ Características } & $\begin{array}{c}\text { Mortalidade Geral } \\
\mathrm{N}=1.013\end{array}$ & $\begin{array}{c}\text { Mortalidade Materna } \\
\mathrm{N}=42\end{array}$ \\
\hline Idade (anos)* & & \\
$10-19$ & 9,9 & 4,8 \\
$20-29$ & 18,4 & 62,0 \\
$30-39$ & 24,1 & 23,8 \\
$40-49$ & 47,6 & 9,6
\end{tabular}

Estado Civil $* *$

$\begin{array}{lrc}\text { solteira } & 50,4 & 59,5 \\ \text { casada } & 28,9 & 26,2 \\ \text { desquitada/viúva/outros } & 5,6 & -\end{array}$

ignorado $\quad 15,0 \quad 14,3$

Ocupação***

$\begin{array}{lrc}\text { doméstica } & 62,0 & 81,0 \\ \text { assalariada } & 17,0 & 14,3 \\ \text { estudante } & 8,4 & 4,8 \\ \text { ignorado } & 12,5 & -\end{array}$

Grau de instrução

nenhum

fundamental

segundo grau

superior

ignorado

$\begin{array}{rr}3,8 & 4,8 \\ 14,1 & 16,7 \\ 2,8 & 2,4 \\ 2,0 & 2,4 \\ 77,4 & 73,8\end{array}$

* $\mathbf{p}<0,0001$

** NS
Tabela 2 - Antecedentes obstétricos dos casos de morte materna. Recife, 1992-1993.

\begin{tabular}{lrr} 
Antecedentes & N & \% \\
\hline Paridade* & & \\
0 & 11 & 32 \\
$1-2$ & 16 & 47 \\
$>3$ & 7 & 21 \\
Com antecedentes de abortos* & 3 & 9 \\
Com antecedentes de cesárea* & 3 & 9 \\
\hline
\end{tabular}

* Faltaram informações a respeito de 8 mulheres

Tabela 3 - Idade gestacional, em semanas, ao fim da gravidez ou no óbito nos casos de morte materna. Recife, 1992-1993.

$\begin{array}{lrr}\text { Idade gestacional } & \mathbf{N} * & \% \\ \text { até } 12 & 5 & 13 \\ 13-20 & 4 & 10 \\ 21-27 & 4 & 10 \\ 28-36 & 9 & 23 \\ 37 \text { ou mais } & 17 & 44 \\ \text { Total } & 39 & 100\end{array}$

* Faltaram informações de 3 mulheres

Tabela 4 - Forma de término da gestação nos casos de mortes maternas. Recife 1992-1993.

\begin{tabular}{|c|c|c|}
\hline Antecedentes & $\mathbf{N}$ & $\%$ \\
\hline Parto & 24 & 57 \\
\hline vaginal & 11 & 46 \\
\hline cesárea & 13 & 54 \\
\hline Aborto & 5 & 12 \\
\hline Ainda gestante & 13 & 31 \\
\hline Total & 42 & 100 \\
\hline
\end{tabular}

Tabela 5 - Distribuição das mortes maternas em hospitais conforme a permanência hospitalar, transferência de outro hospital e tipo de seguro. Recife, 1992-1993.

$\begin{array}{lll}\text { Variáveis } & \mathbf{N} & \%\end{array}$

Tempo de hospitalização (dias)*

$\begin{array}{lrl}1-3 & 21 & 65 \\ 4-6 & 6 & 19 \\ >6 & 5 & 16\end{array}$

Transferência de outro hospital*

$\begin{array}{lrr}\text { sim } & 5 & 16 \\ \text { não } & 27 & 84\end{array}$

Tipo de seguro*

$\begin{array}{lrr}\text { SUS } & 28 & 87 \\ \text { convênios } & 4 & 13\end{array}$

* Faltaram informações sobre a hospitalização em 10 casos. 
Tabela 6 - Distribuição das mortes maternas conforme o momento do óbito em relação ao período da gravidez. Recife, 1992-1993.

\begin{tabular}{lrr}
\hline Momento & N & \% \\
\hline Gestante & 13 & 31 \\
Pós-aborto & 5 & 12 \\
Trabalho de parto/parto & 2 & 5 \\
Puerpério até 42 d & 19 & 45 \\
Puerpério de 43 d - 1 ano & 3 & 7 \\
Total & 42 & 100 \\
\hline
\end{tabular}

Tabela 7 - Distribuição dos recém-nascidos conforme sua vitalidade à época do óbito materno. Recife, 1992-1993.

\begin{tabular}{lrr} 
Vitalidade & N & \% \\
\hline Vivo & 18 & 90 \\
Natimorto & 2 & 10 \\
Total $^{*}$ & 20 & 100 \\
\hline
\end{tabular}

* Sem informações sobre 4 recém-nascidos

\section{Discussão}

Em relação à idade das mulheres que faleceram por complicações da gravidez, parto e puerpério, neste estudo observamos que $62 \%$ das mortes ocorreram em mulheres entre 20 e 29 anos. À primeira vista parece ser esta faixa etária a de maior risco para as mulheres. Contudo, apenas o número de óbitos não pode ser considerado na avaliação do risco de morte materna, pois, provavelmente, nesta faixa etária concentra-se o maior número de mulheres expostas ao risco de engravidar e parir. Isso significaria que, se se fizesse o exercício de calcular a Razão de Mortalidade Materna (RMM) para cada grupo etário, usando no denominador também apenas o número de nascidos vivos referente a cada grupo etário materno, provavelmente encontrar-se-ia para esse grupo a menor RMM, já que contribui proporcionalmente com o maior contingente de nascidos vivos.

No que se refere ao grau de instrução, esta informação parece ter despertado pouco interesse dos que preencheram as declarações de óbito, visto que não estava disponível para a grande maioria dos casos.

O fato de que quatro quintos das mulheres tinham até dois filhos, provavelmente poderia ser explicado pelo relativo maior conhecimento e facilidade de acesso destas pacientes aos métodos anticoncepcionais, principalmente a esterilização tubária. Isto estaria contribuindo para uma mudança no perfil de fecundidade da mulher brasileira, em particular de Recife, tornando menos freqüente a gravidez. Essa consideração está indiretamente de acordo com outro resultado deste estudo de que a hemorragia constituiu apenas a quarta causa dentre as mortes obstétricas diretas ${ }^{6}$. É conhecido que esta geralmente constitui a primeira causa em populações mais desfavorecidas e com maior fecundidade.

Guanto à via de parturição, o presente estudo mostra praticamente o mesmo número de mortes entre mulheres que deram à luz por via vaginal e cesárea, 11 e 13 casos, respectivamente. Entretanto, por ser mais freqüente o parto vaginal, principalmente nos hospitais públicos e em mulheres de classes sociais e econômicas menos favorecidas $^{11,17}$, e com uma estimativa de cesáreas em torno de $25 \%$ de todos os partos para a região Nordeste, ter-se-ia então para os 54.016 nascimentos vivos do município de Recife, 13.504 partos por cesárea. Isso corresponderia a uma razão de mortalidade materna, para esta via de parto, de 96,3 por 100.000 nascidos vivos. Da mesma forma, a RMM para o parto vaginal seria de 27,2/100.000 nascidos vivos. Assim, o risco relativo estimado de morrer quando a parturição foi por via abdominal seria de 3,55 (IC 95\% 1,49-8,47), estatisticamente superior em relação à via vaginal. Este risco mais elevado de morrer pós-parto cesáreo encontra-se de acordo com a maior parte dos autores ${ }^{5,7-9,14}$.

Se não tivesse sido considerada a extensão do período para a conceituação de morte materna para até um ano, cerca de $7 \%$ dos casos não teriam sido identificados, contribuindo também de uma maneira significativa para a subenumeração de mortes maternas já existente ${ }^{1}$.

Neste estudo observamos que apenas 62\% das mulheres que morreram por uma causa relacionada ao ciclo gravídico-puerperal tiveram algum tipo de atendimento pré-natal. Além disso, é importante indagar qual a qualidade do pré-natal oferecido, qual o número de consultas realizadas e em que momento da gestação realizaram-se estas consultas. Sabe-se que o acompanhamento pré-natal adequado é um fator importante na identificação e diminuição dos riscos de morte materna, sobretudo em populações nas quais a prevalência de pré-natal é relativamente baixa ${ }^{3,10,13}$. Foi bastante elevado o número de mulheres que apresentavam patologias prévias à gestação e que se agravaram ou que desenvolveram doenças próprias da gestação. É de se perguntar, assim, se estas mulheres tivessem recebido uma assistência pré-natal adequada, com identificação e controle das patologias associadas, quantas mortes maternas teriam sido evitadas? Todos reconhecem 
a necessidade da assistência pré-natal, mas a simples informação da mulher ou dos familiares de que fez pré-natal não basta para se considerar essa intervenção como satisfatória. É importante a captação precoce da gestante aos serviços de saúde, inclusive fora da gravidez, para avaliação do seu risco reprodutivo e o seguimento adequado, com possibilidade de encaminhá-la para serviços mais capacitados ao atendimento de gestantes de risco. Neste estudo aproximadamente 30\% das mulheres que morreram por complicações da gestação, parto e puerpério tinham uma patologia associada e, em algumas delas, a possibilidade de engravidar deveria ter sido bastante discutida e provavelmente desaconselhada.

\section{SUMMARY}

With the purpose of identifying the social, demographic, pregnancy-related and medical care factors associated with maternal death, this study evaluated all deaths of women aged 10 to 49 years occurring in Recife, Pernambuco, Brazil, during 1992 and 1993. The data were obtained reviewing 1,013 death certificates, with 42 cases of identified maternal deaths. The data of these deaths were complemented with information from medical records, autopsies and also interviews with physicians from the hospitals where the death took place, and with the dead women's relatives. Almost two thirds (62\%) of maternal deaths occurred among women aged 20 to 29 years and more than half of them were single. There was a higher number of deaths among caesarean deliveries than among vaginal ones. The majority of deaths occurred within the first three days of hospitalization and approximately $90 \%$ of hospital charges were sponsored by the National Health System (SUS).

KEY WORDS: Maternal mortality. Pregnancy complications. Abortion. Medical care.

\section{Referências bibliográficas}

1. Albuquerque RM, Cecatti JG, Hardy E, Faúndes A. Mortalidade materna em Recife 1. Avaliação da subenumeração das estatísticas oficiais. Cad Saúde Pública 1997; 13: 59-65.

2. Ariés P. O homem diante da morte. 2. ed. Rio de Janeiro: Francisco Alves; 1990. v. 2. 670p.

3. Ávila MA, Crespo CM, Belfort P. Doença cardíaca e gravidez. Rev Bras Ginecol Obstet 1988; 11: 248-52.
4. Braga LFCO, Nazareno ER, Fanini ML, Carvalho MTW, Soares VMN, Hirata VM. Relatório do Comitê de Morte Materna do Paraná - 1990. Femina 1992; 20: 186-95.

5. Cecatti JG. Análise da mortalidade materna no município de Campinas, no período de 1985 a 1991. Tese Doutorado - Universidade Estadual de Campinas. Campinas, 1992.

6. Cecatti JG, Albuquerque RM, Hardy E, Faúndes A. Mortalidade materna em Recife. Causas de óbitos maternos. Rev Bras Ginecol Obstet 1998; 20:7-11.

7. Costa CFF, Maia WOA, Lomanchinsky G, Camara PAD. Mortalidade materna e perinatal na operação cesariana e no parto transpelviano. Maternidade Prof. Monteiro de Moraes, 1979. J Bras Ginecol 1984; 94: 143-46.

8. Evrard JR, Gold EM. Cesarean section and maternal mortality in Rhode Island. Incidence and risks factors, 1965-1975. Obstet Gynecol 1977; 50: 594-97.

9. Faúndes A, Herrmann V, Cecatti JG. Análise da mortalidade materna em partos cesáreos, no município de Campinas, 1979-1983. Femina 1985; 13: 516-24.

10. Feitosa HN, Moron AF, Born D, Almeida PAM. Mortalidade materna por cardiopatia. Rev Saúde Pública 1991; 25: 443-51.

11. Instituto Brasileiro de Geografia e Estatística. Pesquisa Nacional por Amostra de Domicílio, 1981. Rio de Janeiro: IBGE; 1982.

12. Laurenti R. Marcos referenciais para estudos e investigações em mortalidade materna. Rev Saúde Pública 1988; 22: 507-12.

13. Mauad Filho F, Yazlle MEHD, Carvalheiro CDG, Cunha SP. Prevenção da morte em mulheres grávidas. Considerações gerais. Femina 1989; 17: 365-6.

14. Moldin P, Hokegard KH, Nielsen TF. Cesarean section and maternal mortality in Sweden 1973-1979. Acta Obstet Gynecol Scand 1984; 63: 7-11.

15. Organización Mundial de la Salud. Guia para el estudio de la mortalidad materna en los países en desarrollo. Tasas y causas. Ginebra: WHO; 1997. 1987. 77p. WHO/FHE/87.7.

16. Siqueira AAF, Tanaka ACA, Moron AF, Alvarenga AT, Unglert CVS, Schor N, et al. Estudo da mortalidade materna na região sul do município de São Paulo - Brasil (Análise preliminar). São Paulo, 1991. Série IMCA n ${ }^{\circ} 1$.

17. Tanaka ACA, Siqueira AAF, Bafile PN. Situação de saúde materna e perinatal no estado de São Paulo, Brasil. Rev Saúde Pública 1989; 23: 67-75. 